University of Nebraska - Lincoln

DigitalCommons@University of Nebraska - Lincoln

Faculty Publications from the Department of Electrical \& Computer Engineering, Department Electrical and Computer Engineering

2011

Short-Term Solar Power Prediction Using an RBF Neural Network

Jianwu Zeng

University of Nebraska-Lincoln

Wei Qiao

University of Nebraska-Lincoln, wqiao@engr.unl.edu

Follow this and additional works at: https://digitalcommons.unl.edu/electricalengineeringfacpub

Part of the Electrical and Computer Engineering Commons

Zeng, Jianwu and Qiao, Wei, "Short-Term Solar Power Prediction Using an RBF Neural Network" (2011). Faculty Publications from the Department of Electrical and Computer Engineering. 210.

https://digitalcommons.unl.edu/electricalengineeringfacpub/210

This Article is brought to you for free and open access by the Electrical \& Computer Engineering, Department of at DigitalCommons@University of Nebraska - Lincoln. It has been accepted for inclusion in Faculty Publications from the Department of Electrical and Computer Engineering by an authorized administrator of DigitalCommons@University of Nebraska - Lincoln. 


\title{
Short-Term Solar Power Prediction Using an RBF Neural Network
}

\author{
Jianwu Zeng, Student Member, IEEE, and Wei Qiao, Member, IEEE
}

\begin{abstract}
This paper proposes a radial basis function (RBF) neural network-based model for short-term solar power prediction (SPP). Instead of predicting solar power directly, the model predicts transmissivity, which is then used to obtain solar power according to the extraterrestrial radiation. The proposed model uses a novel two-dimensional (2D) representation for hourly solar radiation and uses historical transmissivity, sky cover, relative humidity and wind speed as the input. Simulation studies are carried out to validate the proposed model for shortterm SPP by using the data obtained from the National Solar Radiation Database (NSRDB). The performance of the RBF neural network is compared with that of two linear regression models, i.e., an autoregressive (AR) model and a local linear regression (LLR) model. Results show that the RBF neural network significantly outperforms the AR model and is better than the LLR model. Furthermore, the use of transmissivity and other meteorological variables, especially the sky cover, can significantly improve the SPP performance.
\end{abstract}

Index Terms--Autoregressive (AR), solar radiation, local linear regression (LLR), neural network, radial basis function (RBF), solar power prediction (SPP)

\section{INTRODUCTION}

$\mathrm{T}_{\mathrm{p}}^{\mathrm{H}}$ HE increasing use of solar power as a source of electricity production has led to increased interest in predicting solar power over short-term horizons. Such a prediction problem is taking on new urgency because solar power prediction (SPP) inaccuracies frequently lead to substantial economic losses and constrain the national expansion of renewable energy [1]. Therefore, it is essential to develop accurate short-term SPP models for operation planning, reserve planning, and peak load matching of power systems [2]. However, the solar radiation reaching the Earth's surface depends upon the climatic conditions of a location [3], which makes the SPP a challenging problem.

There are mainly two categories of SPP methods: physical model-based methods and statistical model-based methods. The physical model is based on physical processes occurring in the atmosphere and influencing solar radiation [4]. It is used to estimate the direct radiation and diffuse radiation with high

This material is based upon work supported by the National Science Foundation under CAREER Award ECCS-0954938 and the Federal Highway Administration under Agreement No. DTFH61-10-H-00003. Any opinions, findings, and conclusions or recommendations expressed in this publication are those of the authors and do not necessarily reflect the view of the National Science Foundation or Federal Highway Administration.

The authors are with the Department of Electrical Engineering, University of Nebraska-Lincoln, Lincoln, NE 68588-0511 (e-mail: wqiao@engr.unl.edu). spatial and temporal resolution [5], clearness index, or cloudiness index (CI) [6]. The CI can be further used to estimate the radiation using the formula in [7]. The physical model does well in medium-term and long-term predictions.

The statistical model is based on time series analysis [8] and does better in short-term prediction. Autoregressive (AR) and autoregressive moving average (ARMA) [9] are among the linear models frequently used in solar and wind power predictions [2], [10]. Nonlinear methods, such as the TakagiSugeno (TS) fuzzy model [11] and wavelet-based methods [12], have been shown superior to linear models. Some studies also indicated that artificial neural networks (ANNs) can achieve a good performance in SPP [12], [13]. These ANNbased models involve modeling of daily or hourly solar radiation, clearness index [14], cloudiness index [15], [16], and effective transmission modeling [17]. Other studies also showed that the SPP using multivariate, such as sun duration, temperature, wind speed, and relative humidity, can achieve much better performance than that using univariate [18]. For example, Rivington [19] predicted solar radiation by using sun duration and air temperature. Ahhi et al. [13] used air temperature, wind speed, sun duration, and relative humidity as the inputs of an ANN to predict solar radiation. However, the effectiveness of using each individual meteorological variable has not been studied yet.

Nevertheless, solar radiations were taken as a 1D time series in most of the existing work, which was turned out to be inferior to a 2D representation [20]. The 2D representation of solar radiations makes it possible to combine image processing methods with nonlinear prediction methods to improve the accuracy of SPP [21]. This paper proposes a radial basis function (RBF) neural network-based method with a $2 \mathrm{D}$ representation of solar radiation and other meteorological variables as the input for SPP. To illustrate the performance of the proposed RBF model, an AR model is served as the reference model and a local linear regression (LLR) model is used for further comparison. Furthermore, the effectiveness of using meteorological variables and different normalization methods are explored. The paper is organized as follows: the SPP models are described in Section II; Section III discusses data preprocessing; simulation studies and conclusions are provided in Sections IV and V, respectively. 


\section{THE SPP MODELS}

\section{A. The AR Model}

Consider an arbitrary time series $X_{t}$, which can be converted to a mean-adjusted time series as follows.

$$
x_{t}=X_{t}-\bar{X}
$$

where $\bar{X}$ is the sample mean of the original time series $X_{i}$; and $x_{t}$ is the mean-adjusted time series. The AR model expresses a time series as a linear function of its past values. The order of the AR model indicates how many past values are used. An AR model with an order of $p, A R(p)$, can be written as:

$$
x_{t}=a_{1} x_{t-1}+a_{2} x_{t-2}+\cdots+a_{p} x_{t-p}+e_{t}
$$

where $\left[x_{t-1}, x_{t-2}, \ldots, x_{t-p}\right]$ are the past values of the time series; $a_{i}$ $(i=1, \cdots, p)$ is the autoregressive coefficient; and $e_{t}$ is noise or error, which is assumed to be a normally distributed random number.

\section{B. The LLR Model}

The use of the LLR model was inspired by the locally high correlations of solar radiations. The idea is that the global nonlinearity of solar radiations can be approximated by multiple local linear models. The LLR differs from the AR model in its time-variant coefficients. These coefficients vary over time when using the LLR for SPP. Let $i$ and $j$ denote the index of hour and day, respectively, then an one-hour 2D linear prediction of solar radiation can be expressed by $\hat{y}_{i, j}=X_{i, j}^{T} a_{i}$, where $X_{i, j}=\left[x_{i-1, j}, x_{i, j-1}, x_{i, j-2}\right]^{\mathrm{T}} ; a_{i}=\left[a_{1}{ }^{(i)}, a_{2}{ }^{(i)}, a_{3}{ }^{(i)}\right]^{\mathrm{T}}$ is the linear coefficient vector, $x_{i j}$ corresponds to the radiation at the $i^{\text {th }}$ hour of the $j^{\text {th }}$ day. Then the error can be estimated as:

$$
e_{i}=\sum_{j=2}^{m}\left(y_{i, j}-X_{i, j}^{T} a_{i}\right)^{2}
$$

where $m$ is the number of training days. The coefficients that minimize the error in (3) can be found from the solution of $\frac{\partial e_{i}}{\partial a_{i}}=0$, which yields the following equation.

$$
\left(\begin{array}{lll}
R_{21}^{(i)} & R_{22}^{(i)} & R_{33}^{(i)} \\
R_{31}^{(i)} & R_{32}^{(i)} & R_{33}^{(i)} \\
\vdots & \vdots & \vdots \\
R_{m 1}^{(i)} & R_{m 2}^{(i)} & R_{m 3}^{(i)}
\end{array}\right) \cdot\left(\begin{array}{l}
a_{1}^{(i)} \\
a_{2}^{(i)} \\
a_{3}^{(i)}
\end{array}\right)=\left(\begin{array}{l}
r_{2}^{(i)} \\
r_{3}^{(i)} \\
\vdots \\
r_{m}^{(i)}
\end{array}\right)
$$

where $R_{j k}^{(i)}$ is the correlation between $x_{i, j}$ and $x_{i, k}$ within the prediction template [21], which is determined by the

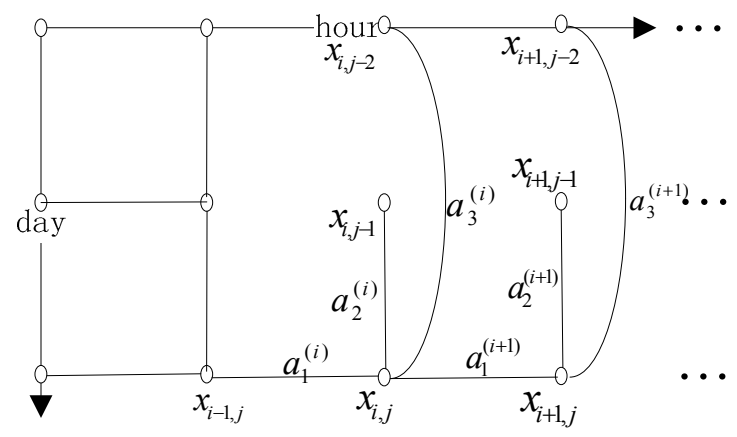

Fig. 1. The one-hour prediction template. correlation analysis in Section III. Fig. 1 shows the one-hourprediction template at the $i^{\text {th }}$ hour, which contains $x_{i, j}, x_{i-1, j}, x_{i, j-1}$ and $x_{i, j-2} . r_{k}^{(i)}$ corresponds to the correlation between the predicted value and each sample in the prediction template. For instance, $r_{1}{ }^{(i)}$ is the correlation between $x_{i, j}$ and $x_{i-1, j} ; r_{2}{ }^{(i)}$ is the correlation between $x_{i, j}$ and $x_{i, j-1}$; etc. Then the coefficients at the $i^{\text {th }}$ hour can be obtained:

$$
a_{i}=\left(R^{(i)}\right)^{+} \cdot r^{(i)}
$$

where $\left(R^{(i)}\right)^{+}$is the pseudo-inverse matrix of $R^{(i)}$. If the index $i$ is changed from one hour to another, then the time-variant coefficient matrix consisting multiple $a_{i}$ can be obtained. It should be noticed that the LLR model becomes the AR model if the coefficients are time-invariant. In other words, the AR model is a special case of the LLR model.

\section{RBF Neural Networks}

RBF neural networks are a class of feed-forward ANNs constructed based on the function approximation theory. Fig. 2 shows the structure of an RBF neural network. It has three functionally distinct layers. The input layer is simply a set of sensory units. The second layer is a hidden layer of sufficient dimension, which performs a nonlinear transformation from the input space to a higher-dimensional hidden-unit space. The third layer performs a linear transformation from the hiddenunit space to the output space. The output of the RBF neural network is given by:

$$
\hat{y}=\sum_{i=1}^{n} w_{i} \phi\left(x, c_{i}, \sigma_{i}\right)+w_{0}
$$

where $n$ is the number of neurons (i.e., RBF units) in the hidden layer; $w_{0}$ is a bias term; $w_{i}$ is the weight between the hidden and output layers; and ( $(\cdot)$ is the activation function in the hidden layer. In this paper, the function $(\cdot)$ is defined as:

$$
\phi\left(x, c_{i}, \sigma_{i}\right)=\exp \left(-\frac{\left\|x-c_{i}\right\|^{2}}{2 \sigma_{i}^{2}}\right)
$$

where $c_{i}$ and $\sigma_{i}$ are the center and width of the RBF function, respectively. The values of $c_{i}$ and $\sigma_{i}$ can be determined by different methods. The simplest method is to randomly choose a subset of the data points as the RBF centers. A more sophisticated approach is to cluster the data into an appropriate number of clusters, whose centers are then used as the centers of the RBF units. In this paper, a local Gaussian mixture model [22] with spherical covariance structure is created to

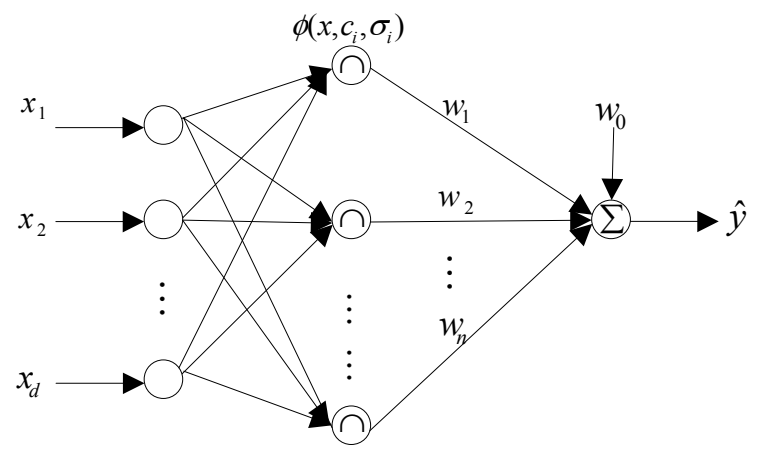

Fig. 2. The structure of an RBF neural network. 
determine the RBF centers by $K$-means clustering algorithm [23]. The Gaussian mixture model is trained by using the Expectation Maximum (EM) algorithm [24]; the resulting centers are then transferred to the RBF neural network.

It has been shown [25] that setting the widths of the RBF functions equal to the variances of the corresponding mixture model tends to give poor results, because the widths are too small and there is insufficient overlap between the RBF functions. In this paper, all the widths are set at the same value; which is proportional to the maximum Euclidean distance, $d_{\text {max }}$, between RBF centers.

$$
\sigma_{i}=k \cdot d_{\max }
$$

where $k$ is a nonnegative scalar, typical value is in the range of $[0.1,0.2][26]$. Given a data set $X$, (6) can be further written as:

$$
\hat{Y}=\Phi \cdot W
$$

where $W=\left[w_{0}, w_{1}, \cdots, w_{n}\right]$ is the vector of the output weights and bias term; and $\Phi$ is the matrix of hidden-layer activations due to the input data $X$. A sum-of-squares error function is defined by

$$
E=\frac{1}{2}\|\hat{Y}-Y\|^{2}
$$

Since this error function is a quadratic function of the vector $W$, pseudo-inverse can be used to determine the optimal $W$ to minimize the value of the error function.

$$
W=\Phi^{+} \cdot Y
$$

where $\Phi^{+}=\left(\Phi^{T} \Phi\right)^{-1} \Phi^{T}$. The Netlab toolbox [25] is used to construct the proposed RBF neural network in simulation studies of the paper.

\section{DATA REPRESENTATION AND PREPROCESSING}

The National Solar Radiation Database (NSRDB) [27] is used to validate the effectiveness of the proposed method. The NSRDB was produced by the National Renewable Energy Laboratory (NREL) in collaboration with other partners. The NSRDB contains 47 variables, including hourly solar radiation and other meteorological data for 1,454 locations in the United States. All the data was recorded from 1991 to 2005. In this paper, the data from San Francisco (Station ID: 724940), Kansas City (Station ID: 724460), and Boston (Station ID: 725090) are selected for simulation studies. The San Francisco data is used in following illustration.

\section{A. 2D Representation}

To visualize the benefits of using $2 \mathrm{D}$ representation, one year data (Jan 1, 2004-Dec 31, 2004) is first considered as a $1 \mathrm{D}$ time series and then as a $2 \mathrm{D}$ image formed in the raster scan form with the columns and rows corresponding to days and hours, respectively. Figs. 3 and 4 show the 1D and 2D representations of the solar radiation data, respectively.

In Fig.3, it is visually difficult to grasp the solar radiation characteristics within a day although the seasonal behavior is obvious. In Fig.4, daily and seasonal behavior of solar radiation can be easily interpreted, where a larger value in the range of $[0,1000]$ indicates a stronger radiation. In winter, the dawn to dusk period is shorter than that of summer. While in

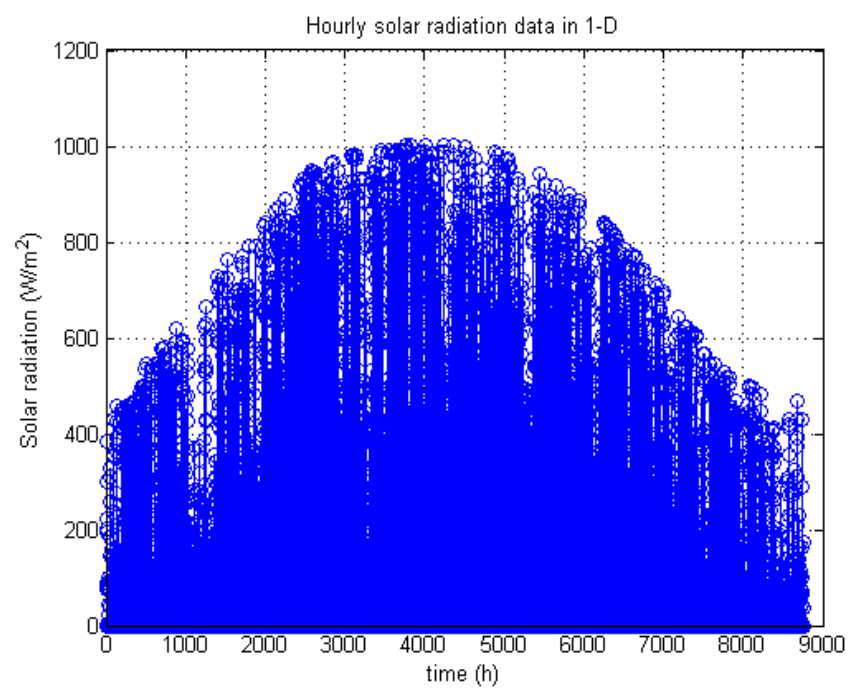

Fig. 3. Hourly solar radiation data in a 1D time plot.

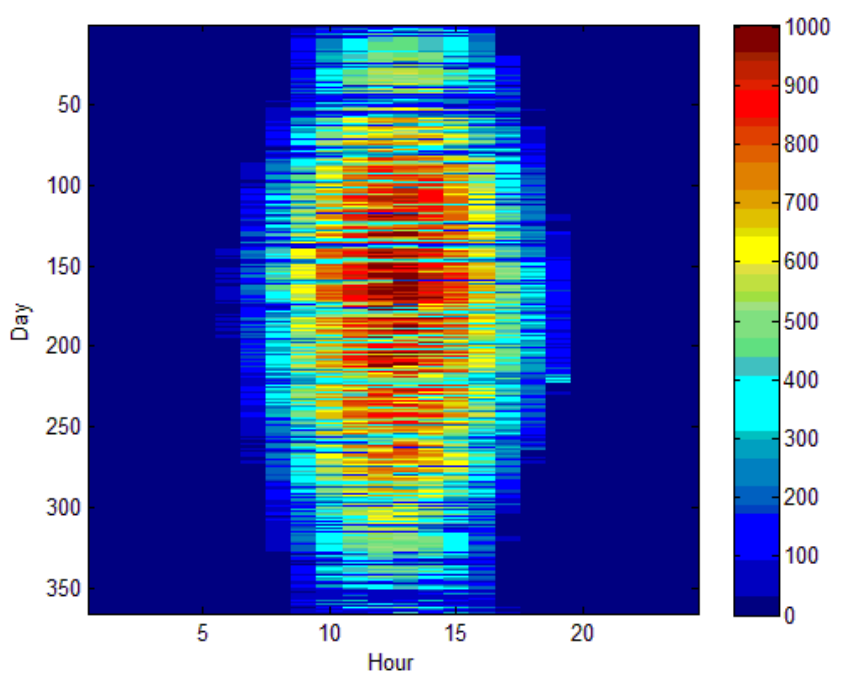

Fig. 4. A 2D image view of the solar radiation data.

summer, radiation at noon achieves the strongest of the whole year. Such a 2D representation provides a significant insight into not only the radiation pattern as a function of time, but also the horizontal and vertical correlations within the $2 \mathrm{D}$ data.

\section{B. Correlation Analysis}

The embedding dimension of the input of the prediction model, i.e., the number of previous data samples used as the input, is determined by the autocorrelation coefficients of the samples.

$$
r_{k}=\frac{1}{(N-k) s^{2}} \sum_{i=k}^{N}\left(x_{i}-\mu\right)\left(x_{i-k}-\mu\right)
$$

where $\mu$ and $s$ are the mean and variance of the samples, respectively; $N$ is the number of samples of the series. Fig. 5 shows a $2 \mathrm{D}$ view of the autocorrelation coefficients of the solar radiation in 2004 .

An important observation from Fig. 5 is that there are strong correlations between the radiations not only in consecutive hours, but also in some hours of consecutive days. 


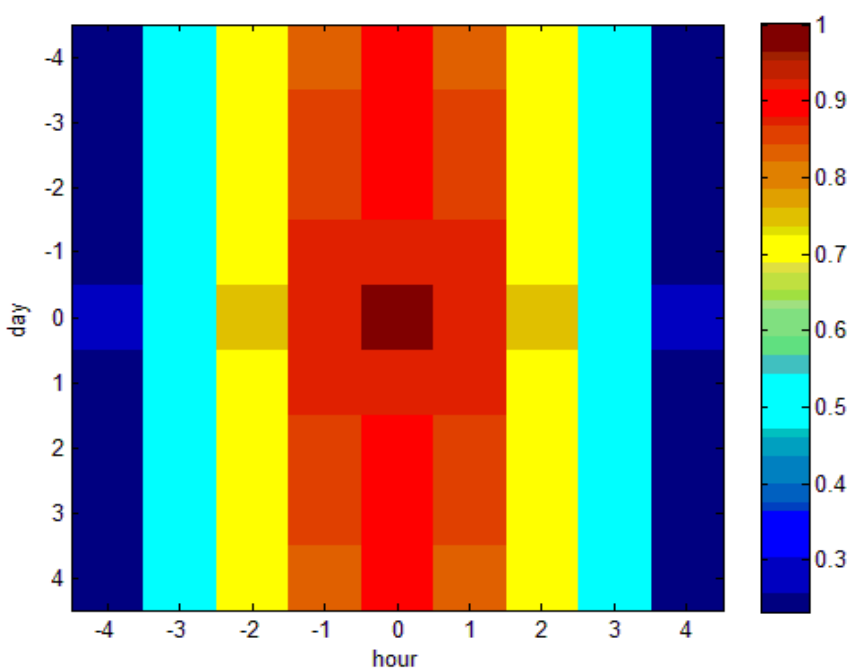

Fig. 5. A 2D view of autocorrelations of the solar radiation.

The correlation between two consecutive days in the same hour is stronger than that between the current hour and 2-hour ahead of the same day. Therefore, when constructing a prediction model, the data from the previous day at the time of prediction, must be used with a higher priority than the data of previous two hours. In this study, the former two days' radiation data at the time of prediction and the data at current time are used as the input of the prediction model.

\section{Normalization}

A data $x$ can be normalized to the range of $[0,1]$ by using the sigmoid function.

$$
y_{n}=\frac{1}{1+\exp \left(-\frac{x_{i}-\mu_{i}}{s_{i}}\right)}
$$

where $\mu_{i}$ and $s_{i}$ are the mean value and standard deviation of the $i^{\text {th }}$ input data, respectively. The sigmoid function can strictly map the original input to the range of $[0,1]$. Moreover, the mean value $\mu_{i}$ and the standard deviation $s_{i}$ make the data translation, rotation, and scale invariant.

Another method of data normalization is based on the concept of transmissivity [17], which is defined as the ratio between the radiation received on the ground surface and the incoming radiation (extraterrestrial radiation) at the top of atmosphere.

$$
\tau=\frac{R_{g}}{R_{e}}
$$

where $\tau$ is the transmissivity; $R_{g}$ and $R_{e}$ are the ground radiation and extraterrestrial radiation, respectively. The extraterrestrial solar radiation $R_{e}$ can be accurately estimated using geometry factors (latitude and longitude), day of the year (DOY), and time of the day (TOD). Therefore, the actual ground radiation can be derived if the transmissivity is known.

The transmissivity takes time variations into account. Therefore, $\tau$ not only reflects the radiation, but also contains certain weather information. A larger $\tau$ is equivalent to a clearer sky, which plays a key role in solar radiation. Due to

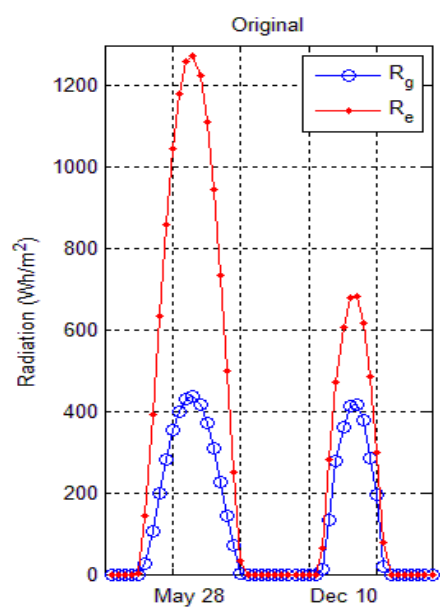

(a)

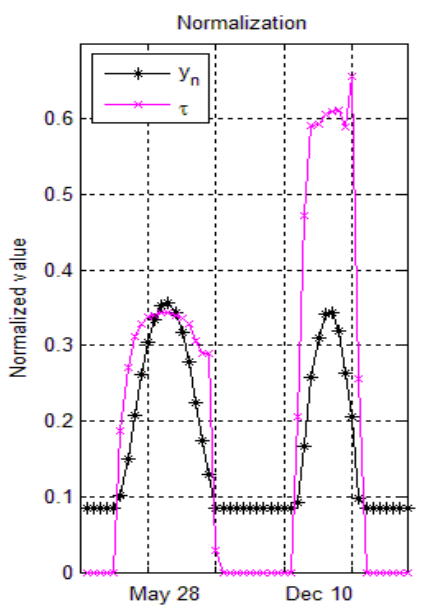

(b)
Fig. 6. Comparison of the two methods for normalization of the radiation data.

its physical meaning, the normalization by transmissivity is superior to that by the sigmoid function. Fig. 6 compares the two methods of normalization for radiation data. Fig. 6(a) is the original radiation on May 28 and Dec. 10, 2005, where the $R_{e}$ curve indicates seasonal variations of the solar radiation. The radiation on May 28 is much stronger than that on Dec. 10. The ground radiation $R_{g}$ curve reflects the effect of the weather condition on solar radiation. For example, May 28 could not be a clear day; otherwise, the ground radiation on May 28 should be much larger than that on Dec 10. Fig. 6(b) shows the normalized values of ground radiation by using the two methods. It can be seen that the sigmoid normalized ground radiation values $\left(y_{n}\right)$ in both days are similar, which fails to 'discover' weather difference. The transmissivity helps 'recognize' the weather condition, which plays an important role in SPP. Therefore, in this paper, the transmissivity is used for normalization of the radiation data; while other variables are normalized by the sigmoid function.

\section{Performance Evaluation}

The mean absolute error (MAE), mean absolute percentage error (MAPE), coefficient of determination $\left(R^{2}\right)$, and correlation coefficient $(\rho)$ are used to evaluate the performance of the SPP models. Definitions are expressed as follows.

$$
\begin{aligned}
& M A E=\frac{1}{N} \sum_{i=1}^{N}\left|\hat{y}_{i}-y_{i}\right| \\
& M A P E=\frac{1}{N} \sum_{i=1}^{N}\left|\frac{\hat{y}_{i}-y_{i} \mid}{y_{i}}\right| \\
& R^{2}=1-\frac{\sum_{i=1}^{N}\left(y_{i}-\hat{y}_{i}\right)^{2}}{\sum_{i=1}^{N}\left(y_{i}-\bar{y}\right)^{2}} \\
& \rho=\frac{\sum_{i=1}^{N}\left(\hat{y}_{i}-\overline{\hat{y}}_{i}\right)\left(y_{i}-\bar{y}\right)}{\sqrt{\sum_{i=1}^{N}\left(\hat{y}_{i}-\overline{\hat{y}}_{i}\right)^{2}} \sqrt{\sum_{i=1}^{N}\left(y_{i}-\bar{y}\right)^{2}}}
\end{aligned}
$$


where $y_{i}$ and $\hat{y}_{i}$ are the observation and the predicted value, respectively; $\bar{y}$ and $\overline{\hat{y}}$ represent the mean values of the observation and prediction, respectively. Smaller values of the MAE and MAPE imply a superior prediction performance of the model. $R^{2}$ is a measure of the global fit of the model. $\rho$ is a measure of linear correlation between two variables.

In order to evaluate the improvement of one model to another, a parameter called skill is defined as follows:

$$
\text { skill }=\frac{\left|e_{0}-e_{1}\right|}{e_{0}} \times 100 \%
$$

where $e_{1}$ and $e_{0}$ are the MAE of the SPP using a new model and the reference model, respectively. A larger skill value indicates more superiority of the new model.

\section{SIMULATION RESULT}

In this section, simulations are carried out for short-term SPP using the NSRDB. The original data is divided into two parts; one is the training set and the other is the testing set, Fig. 7 show the division of the data in one year, where $L_{0}$ is the length of the testing set; $s_{0}$ is the first testing sample; $s=s_{0}+$ $L_{0} / 2$ is the middle point of the testing set; $L_{1}$ is the length of the training set; the range of the training set is $\left[s-L_{1} / 2, s+\right.$ $L_{1} / 2$ ], which has a bilateral symmetric structure. However, if the training and testing data belong to the same year, the range of the training data is $\left[s-L_{1} / 2, s_{0}\right)$. In this study, the testing set contains the data from Sept. 1, 2005 to Sept. 10, 2005, which has moderate numbers of sunny and cloudy days. Then the training data is automatically generated by the method in Fig. 7. In this study, the training set contains the data from July 17 to Oct. 20 in previous years and from July 17 to Aug. 31 in 2005 .

\section{A. Short-Term Prediction}

The training set contains the data from multiple years. Simulations are performed to numerically determine the size of the training set. Fig. 8 shows the MAE and MAPE as functions of the length of the training set (called the training length) for one-hour prediction. As shown in Fig. 8, it is not true that the longer the training length the better the prediction performance. The MAE and MAPE decrease drastically with the increase of the training length up to 7 years. However, after 7 years the MAE and MAPE increase with the training length. Therefore, 7 year is selected as the best training length in the following simulations.

The inputs of all three models include the latest observed solar radiation, radiations at the hour of prediction in the

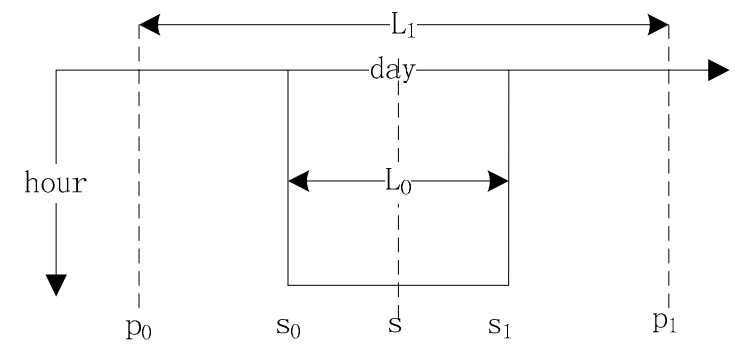

Fig. 7.Training and testing set division.

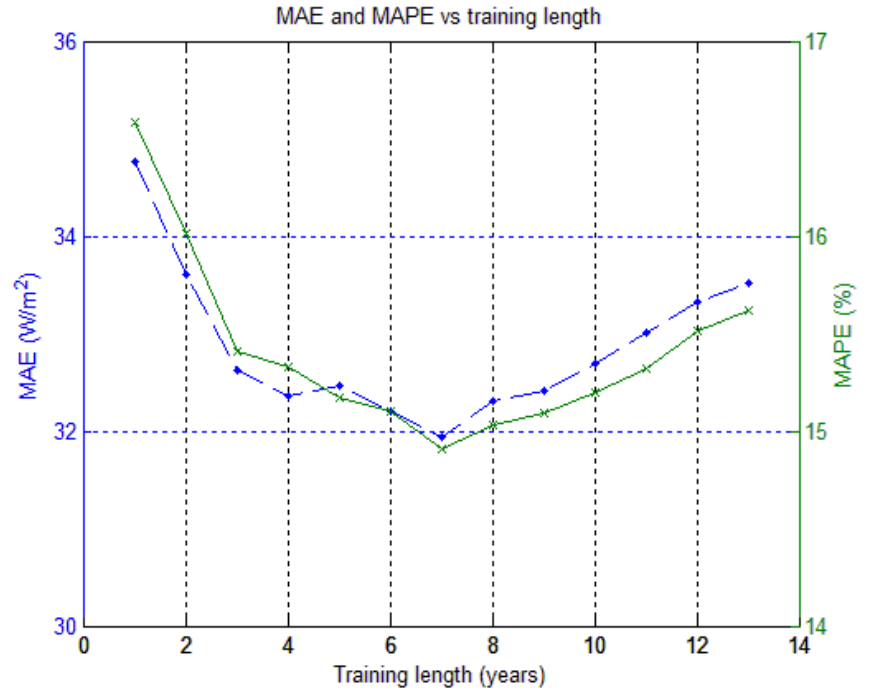

Fig. 8. The MAE and MAPE as functions of the training length.

previous two consecutive days, and the latest meteorological features, including sky cover, wind speed, and relative humidity. In addition, since there is no radiation at night, only the observations from 5 am to $9 \mathrm{pm}$ are used. For one-hour prediction, the radiations from 6 am to $9 \mathrm{pm}$ in a day are predicted. During testing, all of the predicted values are true out-of-sample forecasts, in which only the historical data samples are used. The predicted data is then compared to the actual measured value. The procedure is repeated for the next time step until it runs over the entire testing dataset.

Figs. 9 and 10 show the one-hour-ahead prediction results in Boston using the RBF neural network. As shown in Fig. 9, the RBF neural network works well especially during clear days (the $60^{\text {th }}-150^{\text {th }}$ hours), where the predicted values closely follow the observations. During the clear days, the maximal error is $100 \mathrm{Wh} / \mathrm{m}^{2}$. Large prediction errors mainly occur in overcast days. However, even during the $30^{\text {th }}-60^{\text {th }}$ hours when the weather condition drastically changed, the maximal prediction errors are less than $150 \mathrm{Wh} / \mathrm{m}^{2}$. The error
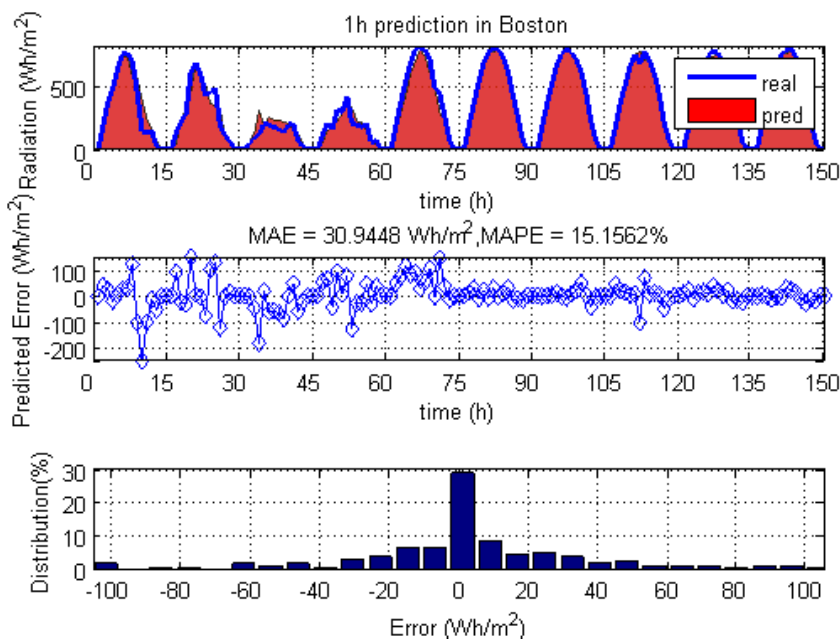

Fig. 9. One-hour-ahead prediction in Boston using the RBF neural network. 


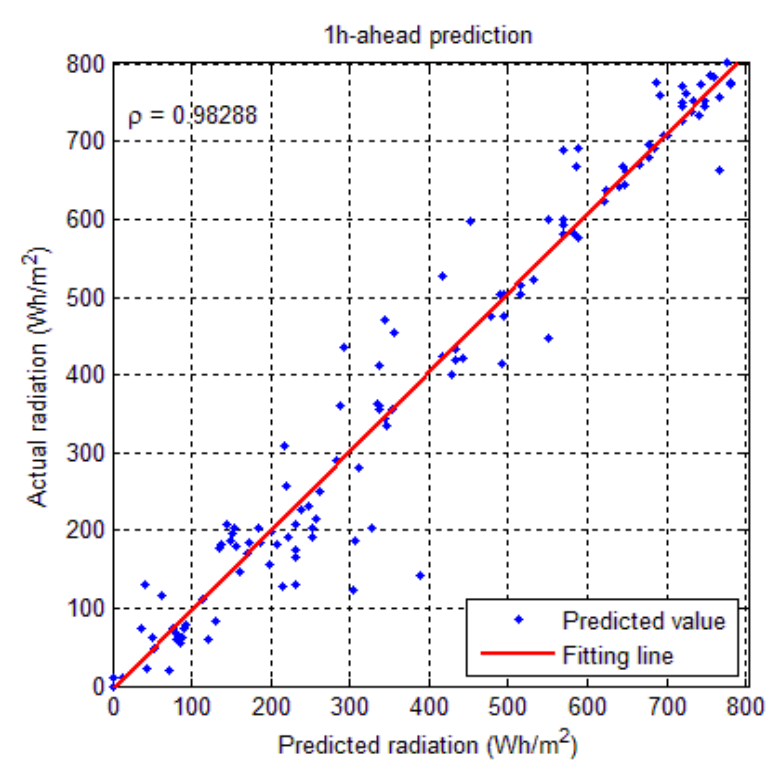

Fig.10. Correlation between the real and predicted solar radiation in Boston.
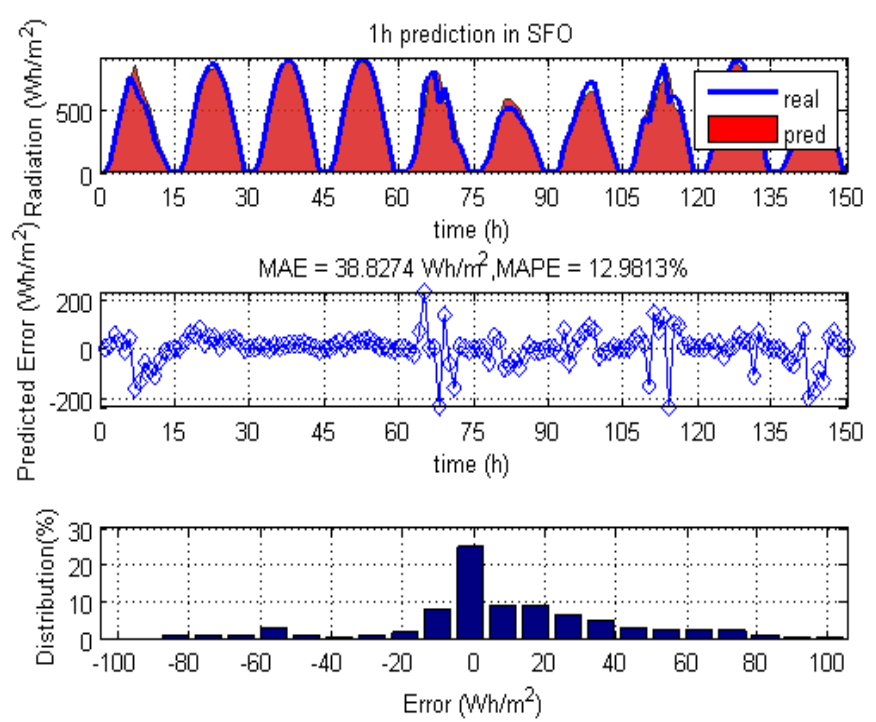

Fig. 11. One-hour-ahead prediction in San Francisco with the RBF neural network.

distribution shows that the majority of prediction errors concentrate in a small range, i.e., approximately $50 \%$ of the predicted errors are less than $20 \mathrm{Wh} / \mathrm{m}^{2}$, which is much smaller than hourly average radiation of $273.26 \mathrm{Wh} / \mathrm{m}^{2}$ in 2004. Fig. 10 shows the collaboration between the real and predicted solar radiation in Boston. As aforementioned, $\rho$ is a measure of linear correlation between two variables. Since $|\rho|$ $<1, \rho=0.98$ corresponds to the slope of the fitting line (1.02), which is close to 45 degrees. Therefore, the predicted values closely match the actual data along the diagonal axis with a narrow scatter, which indicates a successful prediction. Fig. 11 shows the one-hour-ahead prediction in San Francisco by using the RBF neural network. Similarly, it shows an accurate prediction during clear days, i.e., the $15^{\text {th }}-60^{\text {th }}$ hours, in which the errors are less than $20 \mathrm{Wh} / \mathrm{m}^{2}$.
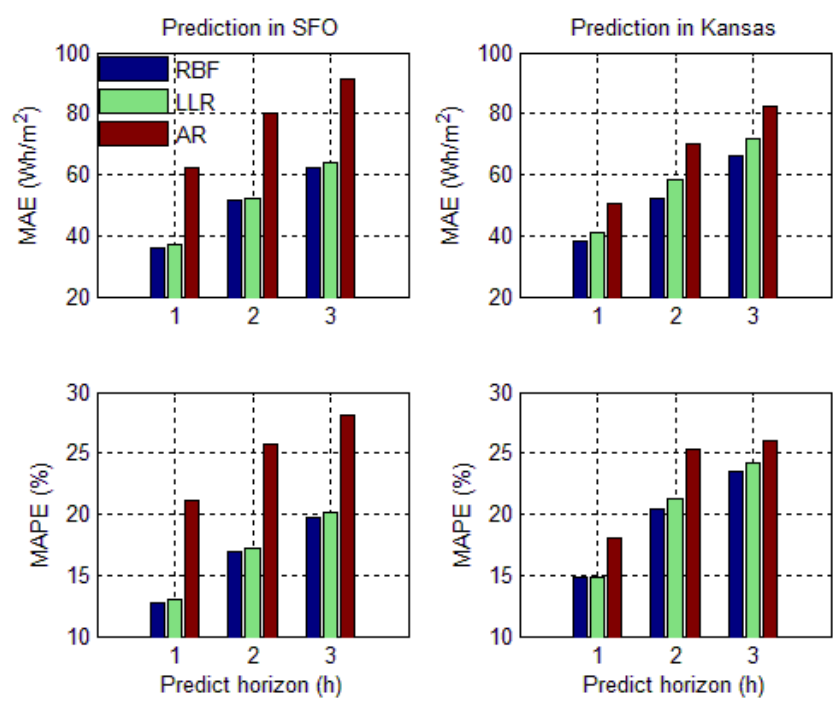

Fig. 12. Comparison of the MAEs and MAPEs of the AR, LLR, and RBF neural network-based prediction models.

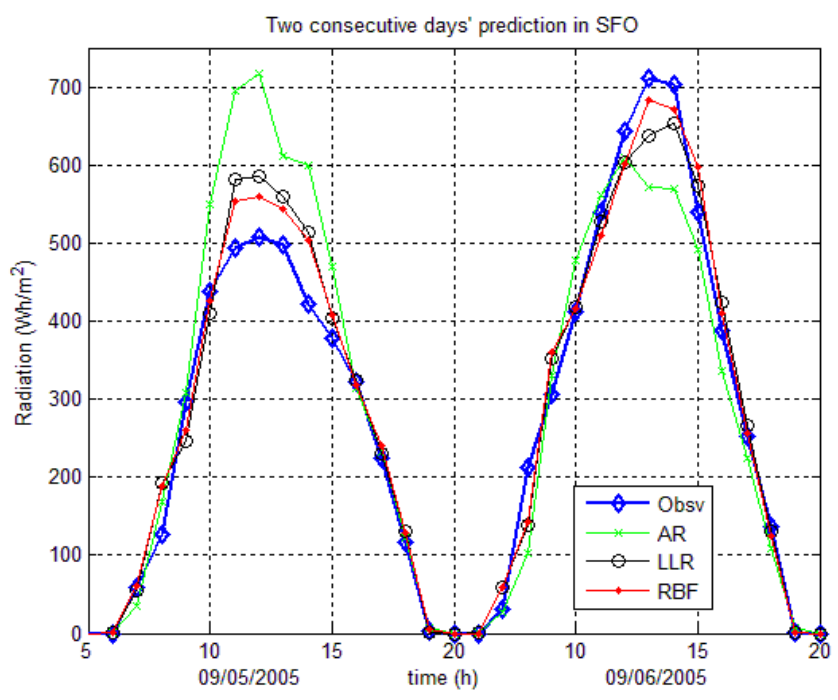

Fig. 13. Comparison of the predicted values from the three models with the observations in two overcast days in SFO.

\section{B. Comparison}

In this paper, the AR model instead of the persistence model in [28] is used as the reference model. Fig. 12 compares the AR, LLR, and RBF neural network-based prediction models using the data in SFO (i.e., San Francisco) and Kansas City. In both sites, the RBF neural network and LLR achieved much better results than the AR model. The success of LLR is due to its multiple local linear models, which capture the global nonlinearity via local linear approximations. On the other hand, the AR model, which uses only one global linear model, fails to predict the solar radiation accurately. However, the LLR is inferior to the RBF neural network, because even within the range of the local linear models, e.g., the model at $12 \mathrm{pm}$, the real data among different days is variable and exhibits nonlinearity. The local regression somehow is too general to approximate the nonlinearity. 
Fig. 13 compares the predicted values from the three models with the observations during two consecutive days in San Francisco. All of the three models can predict with relatively smaller error in the morning and in the afternoon, but not at noon. It seems that the stronger radiation the larger predict error. For example, the predictions do not follow closely the observations form 10 am to $3 \mathrm{pm}$. However, the predictions using the RBF neural network follows the observations more closely than the LLR. All of the three methods yield the predictions smaller than the observations on Sept. 5 but larger predictions on Sept. 6 of 2005 .

\section{Effectiveness Analysis}

Two effectiveness analyses are explored in this study on the use of different normalization methods and meteorological variables. Table I compares the MAEs of the solar radiation prediction using the RBF neural network and two different normalization methods, i.e., the sigmoid function and the transmissivity. The use of transmissivity for radiation normalization reduces the prediction errors to some extent. It is probably because the transmissivity contains certain information that is useful for prediction.

TABLE I: COMPARISON OF MAES (WH/M²) USING DIFFERENT NORMALIZATION METHODS

\begin{tabular}{|c|c|c|c|c|c|c|}
\hline \multirow{2}{*}{$\begin{array}{l}\text { Prediction } \\
\text { Horizon }(\mathrm{h})\end{array}$} & \multicolumn{3}{|c|}{ Sigmoid Function } & \multicolumn{3}{c|}{ Transmissivity } \\
\cline { 2 - 7 } & SFO & Kansas & Boston & SFO & Kansas & Boston \\
\hline 1 & 40.1 & 40.1 & 31.6 & 38.8 & 38.3 & 31.0 \\
\hline 2 & 55.9 & 58.2 & 49.5 & 51.4 & 52.2 & 46.1 \\
\hline 3 & 67.6 & 74.5 & 56.3 & 62.4 & 66.3 & 51.3 \\
\hline
\end{tabular}

Table II compares the prediction results of using the RBF neural network with (the values in brackets) and without meteorological variables in Boston. It indicates that using meteorological information always improves prediction. For instance, when the prediction horizon is 3 hours, the MAE is improved by $11.3 \mathrm{Wh} / \mathrm{m}^{2}$, which corresponds to $18 \%$ improvement over the prediction without meteorological variables. Moreover, MAPE, $\mathrm{R}_{2}$ and $\rho$ are improved by a certain extent as well by using meteorological variables. These are also true in the AR and LLR models. It is obvious that some useful information is provided by these variables.

TABLE II: COMPARISON OF PREDICTIONS WITHOUT (WITH) METEOROLOGICAL VARIABLES IN BOSTON

\begin{tabular}{|c|c|c|c|c|}
\hline Horizon $(\mathrm{h})$ & MAE $\left(\mathrm{Wh} / \mathrm{m}^{2}\right)$ & MAPE $(\%)$ & $\mathrm{R}_{2}$ & $\rho$ \\
\hline 1 & 35.57 & 16.56 & 0.957 & 0.9798 \\
& $(30.95)$ & $(15.16)$ & $(0.962)$ & $(0.981)$ \\
\hline 2 & 53.45 & 23.79 & 0.9142 & 0.956 \\
& $(46.11)$ & $(21.66)$ & $(0.926)$ & $(0.962)$ \\
\hline 3 & 62.59 & 27.05 & 0.892 & 0.945 \\
& $(51.32)$ & $(24.39)$ & $(0.908)$ & $(0.953)$ \\
\hline
\end{tabular}

Another important issue is factor analysis, which explores the importance of the features of the data used for prediction. In this paper, significance of attributes [29] is utilized to quantize the importance of certain features, which include the sky cover, relative humidity, and wind speed. The importance can be evaluated by measuring the effect of removing a feature. In this paper, the three features are removed each time

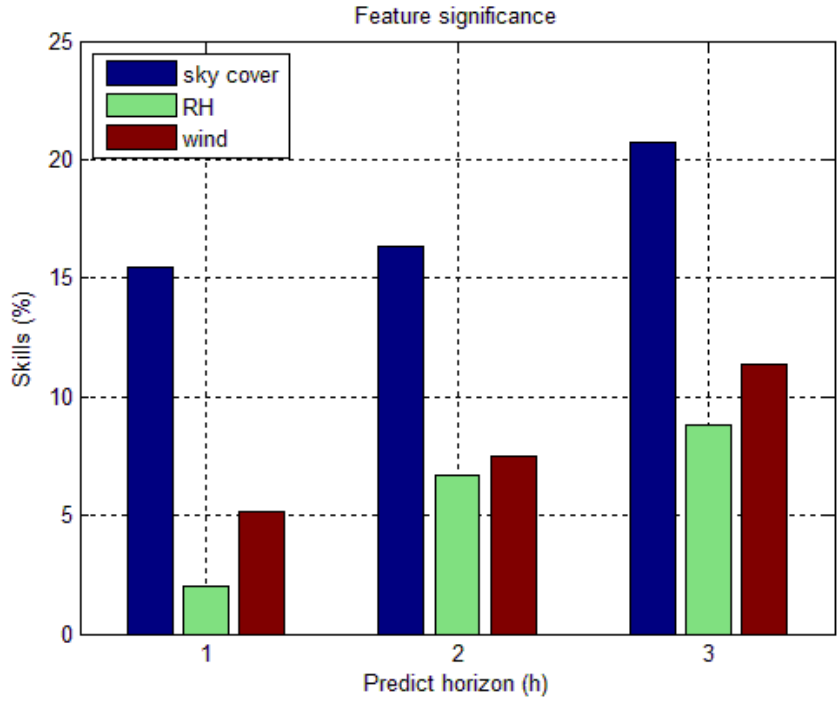

Fig. 14. Comparison of feature significance in Boston

in the radiation predictions in Boston using the RBF neural network; the resulting skills are compared in Fig. 14. The larger the skill is, the more important the feature is. Fig. 14 indicates that the sky cover feature plays a much more important role in prediction than relative humidity and wind speed. This conclusion is consistent with that cloud modulates the distribution of the solar energy reaching the surface, thus changing the energy [30].

\section{CONCLUSIONS}

This paper has presented a RBF neural network-based model, which has been compared with two linear models, for SPP. Simulation studies using the data from the NSRDB at three different sites have yielded several conclusions. First, the proposed RBF neural network-based model has better performance than the AR and LLR models in terms of the prediction accuracy. This is due to the RBF neural network's ability of capturing nonlinear and time-varying nature of the solar radiation data. Second, the proposed model has used a novel 2D representation for hourly solar radiation, which gives more insight into the solar radiation pattern than the regular 1D representation. In addition, since transmissivity contains extra useful information about meteorological features, the normalization with transmissivity has produced lower prediction errors than the sigmoid normalization. Moreover, simulation results have indicated the success of using other meteorological variables to improve the SPP, among which the sky cover is the most important feature. In future work, other nonlinear prediction models will be compared with the RBF neural network-based model.

\section{REFERENCES}

[1] J. W. Hall, "Forecasting solar power with adaptive models - a pilot study," 2010 [Online]. Available: http://www.solardatawarehouse.com/ forecasting\%20solar\%20power.pdf [Accessed on Nov. 18, 2010].

[2] G. Reikard, "Predicting solar radiation at high resolutions: a comparison of time series forecasts", Solar Energy, vol. 83, no. 3, pp. 342-349, March 2009. 
[3] E. Falayi, J. Adepitan, and A. Rabiu, "Empirical models for the correlation of global solar radiation with meteorological data for Iseyin, Nigeria", Physical Sciences, vol. 3, pp. 210-216, Sept. 2008.

[4] V. Badescu, Modeling Solar Radiation at the Earth Surface, first edition, Springer, April, 2008.

[5] R. Chen, E. Kang, X. Ji, J. Yang, and J. Wang, "An hourly solar radiation model under actual weather and terrain conditions: a case study in Heihe river basin", Energy, vol. 32, pp. 1148-1157, 2007.

[6] A. Almakaleh, "New method for energy prediction of solar energy collectors systems in Yemen," in Proc. ISES Solar World Congress 2007: Solar Energy and Human Settlement, pp. 2607-2611, 2009.

[7] F. Kasten and G. Czeplak, "Solar and terrestrial radiation dependent on the amount and type of cloud," Solar Energy, vol. 24, pp. 177-189, 1980.

[8] C. Paolik, C. Voyant, M. Muselli, and M. Nivet, "Solar radiation forecast-ing using ad-hoc time series preprocessing and neural networks," in Proc. $5^{\text {th }}$ International Conference on Emerging Intelligent Computing technology and applications, Ulsan, South Korea, pp. 898-907, 2009.

[9] G. Box, G. M. Jenkins, and G. C. Reinsel, Time Series Analysis: Forecasting and Control, third edition, Prentice-Hall, 1994.

[10] J. L Torres, A. Garcia, M. De Blas, and A. De Francisco, "Forecast of hourly average wind speed with ARMA models in Navarre (Spain)," Solar Energy, vol. 79, pp. 65-77, July 2005.

[11] R. Iqdour and A. Zeroual, "A rule based fuzzy model for the prediction of daily solar radiation," in Proc. International Conference on Industrial Technology, vol. 3, pp.1482-1487, 2004.

[12] G. Gapizzi, F. Bonanno, and C. Napoli, "A wavelet based prediction of wind and solar energy for long-term simulation of integrated generation systems," in Proc. 2010 International Symposium on Power Electronics, Electrical Drives, Automation and Motion (SPEEDAM 2010), pp. 586592, June, 2010.

[13] A. Ahhi, M. Shamisi, and M. Jama, "Prediction of monthly average daily global radiation in Al Ain city - UAE using artificial neural networks," in Proc. $4^{\text {th }}$ Wseas International Conference on Renewable Energy Sources (RES 10), pp. 109-113, Kantaoui, Sousse, Tunisia, May 3-6 2010.

[14] A. Almakaleh, "New method for energy prediction of solar energy collectors systems in Yemen," in Proc. ISES World Congress, pp. $2607-$ 2611, Sept. 2007.

[15] E. M. Crispim, Pedro M. Ferreira, and A. E. Ruano, "Solar radiation prediction using RBF neural networks and cloudiness indices," in Proc. 2006 International Joint Conference on Neural Networks, pp. 2611 2618, Vancouver, BC, Canada, July 16-21, 2006.

[16] R. Perez, S. Wilcox, and D. Renne, "Forecasting solar radiation -preliminary evaluation of an approach based upon the national forecast data base," in Proc. ISES World Congress, Orlando, FL, 2005.

[17] R. Bintanjaand and M. Broeke, "The influence of clouds on the radiations budget of ice and snow surface in Antarctica and Greenland in summer," International Journal of Climatology, vol. 16, pp. 1281-1296, Nov. 1996.

[18] A. Sfetsos and A. H. Coonick, "Univariate and multivariate forecasting of hourly solar radiation with artificial intelligence techniques", Solar Energy, vol.68. no.2, pp. 169-178, 2000.

[19] M. Rivington, G. Bellocchi, K. Matthews, and K. Bachan, "Evaluation of three model estimations of solar radiation at 24 UK stations," Agricultural and Forest Meteorology, vol. 132, pp. 228-243, 2005.

[20] F. O. Hocaoglu, L. N. Gerek, and M. Kurban, "A novel 2-D model approach for the prediction of hourly solar radiation," in Proc. $9^{\text {th }}$ International Work Conference on Artificial Neural Networks, pp. 749756, 2007.

[21] F. O. Hocaoglu, L. N. Gerek, and M. Kurban, "Hourly solar radiation forecasting using optimal coefficient 2-D linear filters and feed-forward neural networks," Solar Energy, vol. 82, pp. 714-726, 2008.

[22] C. E. Rasmussen, "The infinite Gaussian mixture model," in Proc. Advances in Neural Information Processing Systems 12, pp. 554-560, 2000.

[23] J. MacQueen, "Some methods for classification and analysis of multivariate observations," in Proc. $5^{\text {th }}$ Berkeley Symposium on Mathematical Statistics and Probability, vol. 1, pp. 281-297, 1967.

[24] A. P. Dempster, N.M. Laird, and D.B. Rubin, "Maximum likelihood from incomplete data via the EM algorithm," Journal of the Royal Statistical Society, Series B, vol. 39, no. 1, pp. 1-38, 1977.

[25] I. T. Nabney, Netlab Algorithms for Pattern Recognition, Verlag, Springer press, 2002, pp. 191-198.
[26] J. Shi and J. Malik, "Normalized cuts and image segmentation," IEEE Trans. on Pattern Analysis and Machine Intelligence, vol. 22, no. 8, pp. 888-905, Aug. 2000.

[27] National Renewable Energy Laboratory, "National solar radiation database 1991-2005 update: user's manual," National Renewable Energy Laboratory, Tech. Rep. TP-581-41364, April 2007.

[28] L. Martin, L.F. Zarzalejo, J. Polo, A. Navarro, R. Marchante, and M. Cony, "Prediction of global solar irradiance based on time series analysis: application to solar thermal power plants energy production planning," Solar Energy, vol. 84, pp. 1772-1781, 2010.

[29] J. Komorowski, L. Polkowski, and A. Showron, "Rough sets: a tutorial," $1998 \quad$ [Online]. Available: http://folli.loria.fr/cds/1999/library/pdf/skowron.pdf [Accessed on Nov. 21, 2010].

[30] V. Ramanathan, "The role of earth radiation budget studies in climate and general circulation research," Journal of Geophysical Research, vol. 92, no. D4, pp. 4075-4095, 1987.

\section{BIOGRAPHIES}

JianwuZeng ( $\left.S^{\prime} 10\right)$ received the B.Eng. degree in electrical engineering from Xi'an University of Technology, Xi'an, China, in 2004, and the M.S. degree in control science and engineering from Zhejiang University, Hangzhou, China, in 2006. Currently, he is pursuing the Ph.D. degree at the University of Nebraska-Lincoln, USA.

From 2006 to 2008, he was an Electronic Engineer with Santak Electronic (Shenzhen) Co., Ltd., Shenzhen, China, where he was involved in research and development on soft switching and DC-DC converters. His research interests include power electronics and renewable energy.

Wei Qiao (S'05-M'08) received the B.Eng. and M.Eng. degrees in electrical engineering from Zhejiang University, Hangzhou, China, in 1997 and 2002, respectively, the M.S. degree in high performance computation for engineered systems from Singapore-MIT Alliance (SMA), Singapore, in 2003, and the $\mathrm{Ph} . \mathrm{D}$. degree in electrical engineering from Georgia Institute of Technology, Atlanta, in 2008.

From 1997 to 1999, he was an Electrical Engineer with China Petroleum \& Chemical Corporation (Sinopec). Since 2008, he has been an Assistant Professor of Electrical Engineering with the University of NebraskaLincoln. His research interests include renewable energy systems, smart grids, power system control and optimization, condition monitoring and fault diagnosis, energy storage, power electronics, electric machines, and highperformance computation for electric power and energy systems. He is the author or coauthor of 3 book chapters and more than 60 papers in refereed journals and international conference proceedings.

Dr. Qiao is an Associate Editor of the IEEE Transactions on Industry Applications, the Chair of the Sustainable Energy Sources Subcommittee of the IEEE Power Electronics Society, and the Chair of the Task Force on Intelligent Control for Wind Plants of the IEEE Power \& Energy Society. He was the Technical Program Cochair and Finance Cochair of the 2009 IEEE Symposium on Power Electronics and Machines in Wind Applications. He has organized and chaired several special and regular sessions at international conferences.

Dr. Qiao was the recipient of a 2010 NSF CAREER Award and the 2010 IEEE Industry Applications Society Andrew W. Smith Outstanding Young Member Award. 